\title{
SUPERCOMPUTING '90: COMPUTER-CHESS TESTING AND PROGRAMMING SESSION
}

\author{
John Stanback \\ November 14, 1990 \\ New York
}

This year three papers were presented at the technical session held in conjunction with the $21^{\text {st }}$ NACCC tournament. A brief summary of the presentations is given here.

The first paper, titled A Taxonomy of Concepts for Evaluating Chess Strength by Hans Berliner, Danny Kopec, and Ed Northam was presented by Danny Kopec [1, pp. 336-343]. He noted that while leading computer chess programs are quite strong tactically and have achieved high ELO ratings, it is recognized that they are deficient in chess knowledge, i.e., performance does not indicate comprehension. The Chess Master understands what is required in particular chess positions and knows when to break the rules. How can we quantify knowledge of abstract chess concepts such as king safety, space, and connectivity so as to test the computers' understanding? Although previous suites of test positions have shown good correlation between the strength of a chess program and its ability to solve problems, they have not been comprehensive and positions were not well selected for determining the presence of specific chess knowledge. A taxonomy of more than 50 chess concepts covering opening themes, tactical and strategical middle-game themes, and domain-specific endgame knowledge has been developed, and test positions involving these concepts are currently being selected. A few of these positions were presented at this session. It is expected that a suite of over 200 test positions will be made available in machine readable form.

The second speaker was Lewis Stiller who presented a paper titled Group Graphs and Computational Symmetry on Massively Parallel Architecture [1, pp. 344-353]. Lewis described how the retrograde analysis procedure was implemented on the parallel architecture of a $64 \mathrm{~K}$ processor Connection Machine to achieve immense speedups over conventional techniques on a single processor. A typical analysis which takes 100 seconds on the CM-2 might take over 10 hours on a single-processor machine. He explained how the exploitation of symmetry reduces the storage requirements of a five-piece chess endgame database from about one gigabyte to about 120 megabytes. He believes the six-piece endgames are achievable, with a storage requirement of about six gigabytes. The explanation of the complex coding scheme used was quite technical and not understood by this reviewer. Details can be obtained by contacting the author (Unix mail address: stiller@cs.bu.edu).

The third speaker was Jonathan Schaeffer who gave a presentation on the computer-checkers program CHINOOK. This impromptu talk replaced the planned presentation Chess and Supercomputers: Details about optimizing Cray Blitz by Robert Hyatt and Harry Nelson [1, pp. 354-363]. Jonathan began by giving a brief history of computer-checkers programs, in particular the Samuel program developed in the early 1960s and the Duke University program developed in the 1970s. He described the progress of the CHINOOK program which was begun in 1989 at the University of Alberta. This year it placed first in the Mississippi State Checkers Championship and second in the US Open Checkers Championship. The program has defeated several of the top 10 players in the world and is currently ranked second in the world behind champion Marion Tinsley. The first goal is to defeat the human World Champion and it appears that CHINOOK will get a chance to do so in a match with Tinsley next spring. A longer term goal is to solve the game of checkers. Jonathan then described the basic components of CHINOOK. A brute-force alpha-beta search is employed which achieves depths of 15-27 ply. Checkers knowledge consists of a linear combination of about 20 evaluation terms (each with four 'phases') which are manually tuned by a master checkers player. Endgame databases currently generated cover endgames up to six pieces and contain over ten billion positions. An eight-piece database will consist of more than 400 billion positions and would take years of CPU time using the current retrograde-analysis procedure. It is felt that with the eight-piece database in place it is likely that CHINOOK will solve the game of checkers. Current research involves techniques for generating and storing these huge databases, determining an algorithm for choosing the 'best' move from several of which the program knows they all will draw, and developing a method for automatic tuning of the evaluation function.

[1]. Proceedings Supercomputing '90, New York, November 1990; IEEE Computer Science Press, Los Alamitos, California. 